\title{
Porphyrin Immobilized Nanographene Oxide for Enhanced and Targeted Photothermal Therapy of Brain Cancer
}

Siheng Su, Jilong Wang, Evan Vargas, Junhua Wei, Raul Martínez-Zaguilán, Souad R. Sennoune, Michelle L. Pantoya, Shiren Wang, Jharna Chaudhuri and Jingjing Qiu*

List:

Figure S1: Photograph of set-up of agar gel irradiation......................... S2

Figure S2: Photograph of set-up of photothermal therapy on a mouse............... S2

Figure S3: X-ray photoelectron spectroscopy profile of GO........................ 3

Figure S4: Fluorescence spectra of GO, free Porphyrin and GO/Porphyrin mixture......S3

Figure S5: Dynamic light scattering (DLS) measurements of PNG .................S4

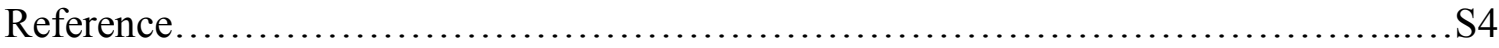




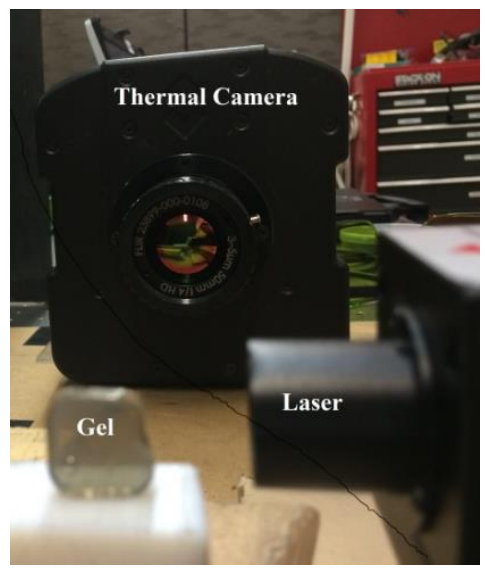

Figure S1: Photograph of set-up of agar gel irradiated by $808 \mathrm{~nm}$ laser and recorded by IR camera

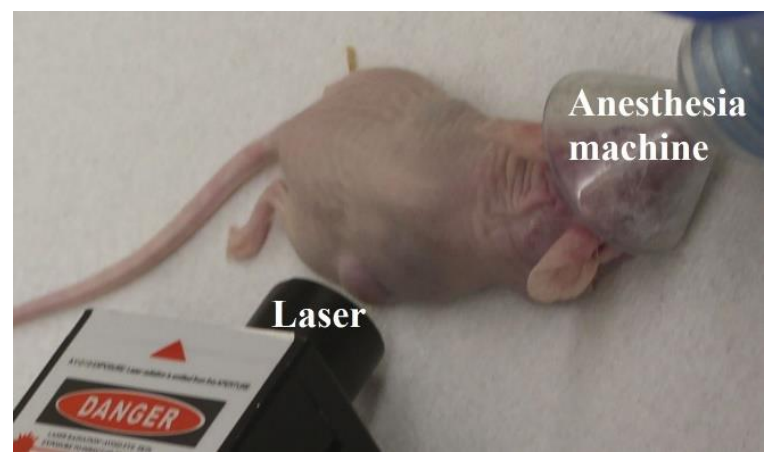

Figure S2: Photograph of set-up of photothermal therapy on a mouse 

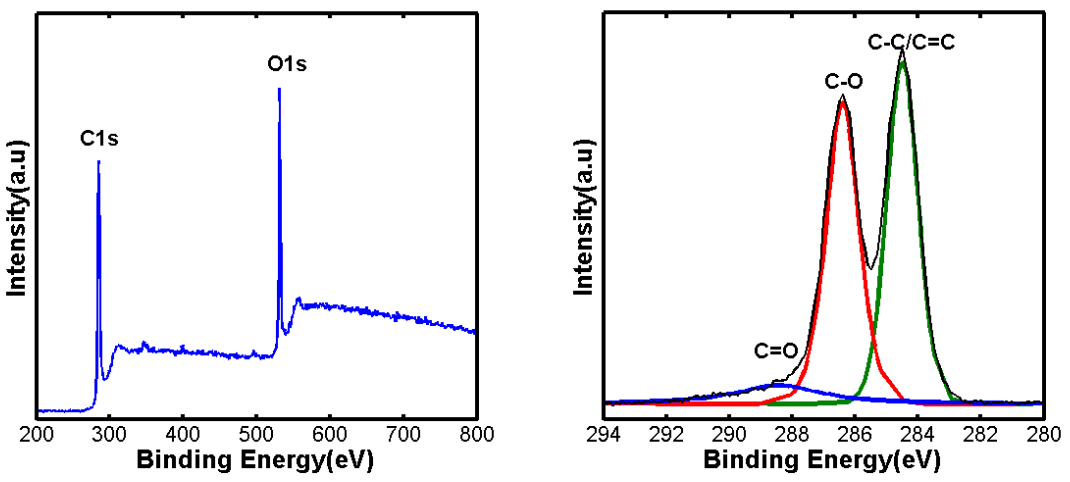

Figure S3: (a) XPS survey spectrum of GO, and (b) high-resolution XPS C1s spectra of GO. The oxygenated groups in GO are demonstrated by XPS, which shows epoxy/hydroxyl groups $(\mathrm{C}-\mathrm{O})$ at $286.4 \mathrm{eV}$, carboxyl groups $(\mathrm{C}=\mathrm{O})$ at $288.5 \mathrm{eV}$, while $\mathrm{C}$ $\mathrm{C} / \mathrm{C}=\mathrm{C}$ presents at $284.5 \mathrm{eV}^{1-2}$.

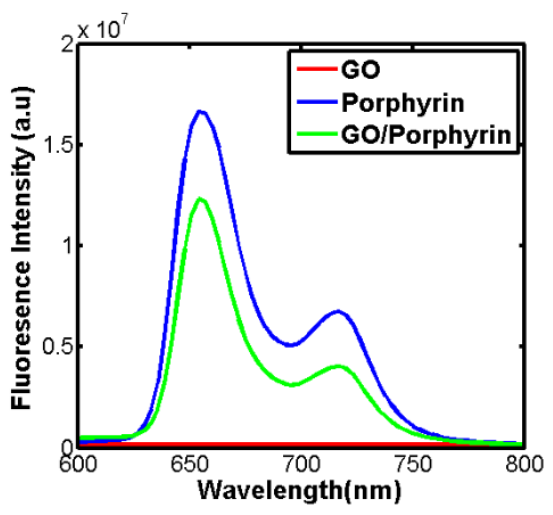

Figure S4: Fluorescence spectra of GO, free Porphyrin and GO/Porphyrin mixture at $0.06 \mathrm{mg} / \mathrm{ml}$ porphyrin equivalent under $500 \mathrm{~nm}$ excitation. It is shown that fluorescence of porphyrin was quenched by GO, resulting from the direct contact between porphyrin and $\mathrm{GO}$, suggesting the energy transfer from porphyrin to GO. 


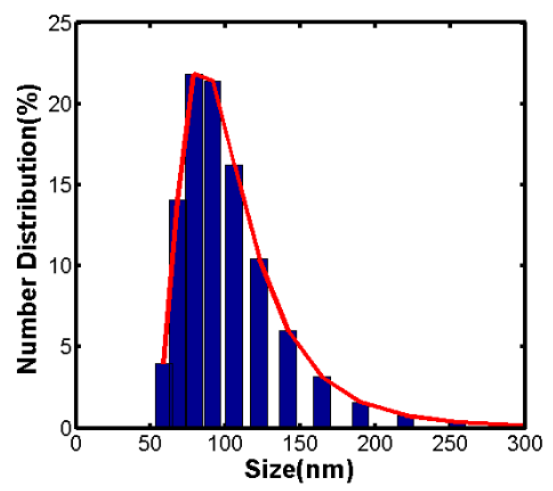

Figure S5: Dynamic light scattering (DLS) measurements of aqueous dispersions of PNG. Mean size is $78 \mathrm{~nm}$.

\section{References}

1. Xiao, F.; Yang, S.; Zhang, Z.; Liu, H.; Xiao, J.; Wan, L.; Luo, J.; Wang, S.; Liu, Y., Scalable synthesis of freestanding sandwich-structured graphene/polyaniline/graphene nanocomposite paper for flexible all-solid-state supercapacitor. Scientific reports 2015, 5, 9359.

2. Zhang, Y.; Yuan, X.; Wang, Y.; Chen, Y., One-pot photochemical synthesis of graphene composites uniformly deposited with silver nanoparticles and their high catalytic activity towards the reduction of 2-nitroaniline. Journal of Materials Chemistry 2012, 22 (15), 7245-7251. 